\title{
Anti-Diabetic Effect of Pectinase-Processed Ginseng Radix (GINST) in High Fat Diet-Fed ICR Mice
}

\author{
Hai Dan Yuan, Hai Yan Quan, Mi Song Jung, Su Jung Kim, Bo Huang, Do Yeon Kim, \\ and Sung Hyun Chung* \\ Department of Life and Nanopharmaceutical Science, Graduate School of Kyung Hee University, Seoul 130-701, Korea
}

In the present study, we investigate anti-diabetic effect of pectinase-processed ginseng radix (GINST) in high fat diet-fed ICR mice. The ICR mice were divided into three groups: regular diet group, high fat diet control group (HFD), and GINSTtreated group. To induce hyperglycemia, mice were fed a high fat diet for 10 weeks, and mice were administered with $300 \mathrm{mg} /$ $\mathrm{kg}$ of GINST once a day for 5 weeks. Oral glucose tolerance test revealed that GINST improved glucose tolerance after glucose challenge. Compared to the HFD control group, fasting blood glucose and insulin levels were decreased by $57.8 \%(p<0.05)$ and $30.9 \%(p<0.01)$ in GINST-treated group, respectively. With decreased plasma glucose and insulin levels, the insulin resistance index of the GINST-treated group was reduced by $68.1 \%(p<0.01)$ compared to the HFD control group. Pancreas of GINST-treated mice preserved a morphological integrity of islets and consequently having more insulin contents. In addition, GINST up-regulated the levels of phosphorylated AMP-activated protein kinase (AMPK) and its target molecule, glucose transporter 4 (GLUT4) protein expression in the skeletal muscle. Our results suggest that GINST ameliorates a hyperglycemia through activation of AMPK/ GLUT4 signaling pathway, and has a therapeutic potential for type 2 diabetes.

Keywords: Panax ginseng, Pectinase-processed ginseng radix, High fat diet, AMP-activated protein kinase, Glucose transporter type 4

\section{INTRODUCTION}

Type 2 diabetes, a complex metabolic disease, is increasing rapidly with higher rates of morbidity and mortality $[1,2]$. It is characterized by insulin resistance and hyperglycemia, which lead to chronic complications such as diabetic nephropathy, neuropathy, and retinopathy [3]. Insulin resistance is a key defect underlying type 2 diabetes [4]. Insulin resistance in skeletal muscle is manifested by decreased insulin-stimulated glucose uptake and results from impaired insulin signaling and multiple postreceptor intracellular defects including impaired glucose transport, glucose phosphorylation, and reduced glucose oxidation and glycogen synthesis [5]. Therefore, numer-

(c) This is an Open Access article distributed under the terms of the Creative Commons Attribution Non-Commercial License (http://creativecommons.org/licenses/by-nc/3.0/) which permits unrestricted non-commercial use, distribution, and reproduction in any medium, provided the original work is properly cited. ous studies have focused on development of therapeutic agents able to maintain normal levels of blood glucose by increasing glucose clearance in peripheral tissues such as skeletal muscle and adipose tissue $[6,7]$.

Ginseng is a commonly used traditional Chinese medicine. The root of ginseng has been used for treatment of various diseases in Asian countries for thousand years. Modern pharmacological research has found that ginseng exerts anti-diabetic, anti-cancer, anti-inflammatory, and anti-oxidant effects [8-11]. The main bioactive components in ginseng are ginsenosides. Hitherto, ginsenosides $\mathrm{Rb}_{1}, \mathrm{Rg}_{1}, \mathrm{Rg}_{3}, \mathrm{Rh}_{2}, \mathrm{Re}$, and IH-901 have been docu-

Received 24 Feb. 2011, Revised 21 May. 2011, Accepted 21 May. 2011

*Corresponding author

E-mail: suchung@khu.ac.kr

Tel: +82-2-961-0373, Fax: +82-2-957-0384 
mented in many studies and found the anti-diabetic effects of ginsenosides [12-16]. To develop safer and more effective anti-diabetic agents from ginseng radix, ginseng radix was treated with pectinase enzyme and obtained pectinase-processed ginseng radix (GINST). Here, we investigate the anti-diabetic activity and action mechanism of GINST using ICR mice fed a high fat diet.

\section{MATERIALS AND METHODS}

\section{Chemicals}

Pectinase was purchased from the DSM food specialties (ZAE La Baume, Servian, France). Antibodies against AMP-activated protein kinase (AMPK), phosphoAMPK, acetyl-CoA carboxylase (ACC), phospho-ACC, glucose transporter 4 (GLUT4) were from Cell Signaling Technology (Beverly, MA, USA), and anti-actin, antiinsulin, and anti-goat IgG-HRP were from Santa Cruz Biotechnology (Santa Cruz, CA, USA). Protein extraction and western blotting detection kits were from Intron Biotechnology Inc. (Beverly, MA, USA). Bio-Rad protein assay kit was from Bio-Rad Laboratories (Hercules, CA, USA). Polyvinylidine difluoride membranes was from Millipore (Bedford, MA, USA), Other reagents and chemicals were of analytical grade.

\section{Preparation of pectinase-processed ginseng radix}

GINST was obtained from Ilhwa Co. Ltd. (Guri, Korea). Briefly, the ginseng radix (containing with $30 \%$ to $40 \%$ moisture) was extracted from $40 \%$ to $50 \%$ ethanol and concentrated with a speed-vac, then incubated with an enzyme solution containing pectinase at $55^{\circ} \mathrm{C}$ for $24 \mathrm{~h}$.

\section{Analysis of ginsenosides in pectinase-processed ginseng radix}

An Acquity liquid chromatograph (Waters, Milford, MA, USA) equipped with gradient pump, autosampler, and diode array detection was used. An Acquity UPLC BEH C18 reversed-phase column $(100 \times 1.0 \mathrm{~mm}$, i.d., 1.7 $\mu \mathrm{m}$ ) was used. The mobile phase consisted of water (solvent $\mathrm{A}$ ) and acetonitrile (solvent $\mathrm{B}$ ). The gradient elution was used as follow: $0-3 \mathrm{~min}, 5 \% \mathrm{~B} ; 10 \mathrm{~min}, 15 \% \mathrm{~B} ; 12$ $\min , 30 \% \mathrm{~B} ; 15 \mathrm{~min}, 35 \% \mathrm{~B} ; 20 \min 60 \% \mathrm{~B}$. The column temperature was kept constant at $35^{\circ} \mathrm{C}$, and the flow rate was $0.5 \mathrm{~mL} / \mathrm{min}$.

\section{Animals}

Five-week-old ICR mice were purchased from Orient Bio (Seoul, Korea). All animals were acclimatized to the laboratory environment for 1 week before the experi- ment. Mice were allowed to freely access to drinking water and food under constant room temperature $\left(22 \pm 2^{\circ} \mathrm{C}\right)$ and humidity $(50 \pm 10 \%)$ conditions with an automatic $12 \mathrm{~h}$ light and dark cycle and experimental protocol was approved by the Institutional Animal Ethics Committee of the Kyung Hee University. Mice were randomly divided into three groups as following; group fed a regular $\operatorname{diet}(\mathrm{RD})$, group fed a high fat diet (HFD; Research diet, New Brunswick, NJ, USA), group treated with $300 \mathrm{mg}$ / $\mathrm{kg}$ of GINST (GINST). In the treatment group, mice were orally administered once a day for $5 \mathrm{wk}$ after freely access to HFD for $5 \mathrm{wk}$.

\section{Oral glucose tolerance test}

At the end of the experiment, the ICR mice were fasted for $12 \mathrm{~h}$ prior to the experiment. Glucose $(1.5 \mathrm{mg} /$ $\mathrm{kg}$ ) was orally administered at $0 \mathrm{~min}$, and the blood was withdrawn from the orbital venous plexus at $0,30,60$ and 90 min after glucose administration. Plasma level was determined by the glucose oxidase method [13].

\section{Determination of serum parameters}

At the end of treatment, mice were anaesthetized by diethyl ether and blood samples were collected by cardiac puncture. Blood samples were centrifuged at $3,000 \times g$ for $15 \mathrm{~min}$ at $4^{\circ} \mathrm{C}$, and plasma glucose level was determined using commercial kit (Stanbio Laboratory, Boerne, TX, USA) and automatic analyzer (SMARTLAB, Mannheim, Germany). The plasma insulin concentration was determined using a mouse insulin enzyme immunoassay kit (Shibayagi, Gunma, Japan).

\section{Histological analysis}

For hematoxylin-eosin staining, the pancreas tissue was removed and fixed in 10\% neutral buffered formalin. The tissues were subsequently embedded in paraffin and sectioned with $5 \mu \mathrm{m}$ thickness (Leica, Wetzlar, Germany), and stained with hematoxylin-eosin for microscopic assessment (Olympus, Tokyo, Japan). To examine the insulin contents in pancreas, immunohistochemistry technique was used. The sections were deparaffinized in $x y-$ lene and rehydrated through a graded ethanol series. Antigen retrieval was performed by $0.1 \%$ trypsin. To block nonspecific binding of immunoglobulin, the sections were incubated with normal serum blocking solution for $30 \mathrm{~min}$ at room temperature. Goat anti-insulin $\operatorname{IgG}(1: 75)$ were applied overnight at $4^{\circ} \mathrm{C}$, then the tissue sections were incubated with donkey anti-goat IgG-HRP (1:200) for $30 \mathrm{~min}$ at room temperature. Positive control was visualized DAB peroxide substrate solution for 5 to $10 \mathrm{~min}$, 
and tissues were counterstained with hematoxylin.

\section{Western blot analysis}

Total protein extracts were prepared using a protein extraction kit and insoluble protein was removed by centrifugation at 13,000 g for $20 \mathrm{~min}$. Protein concentrations in cell lysates were measured using a Bio-Rad protein assay kit. For Western blotting, $40 \mu \mathrm{g}$ of protein was separated by $8 \%$ SDS-polyacrylamide gel electrophoresis and transferred to polyvinylidene difluoride membranes. The membrane was further incubated with the indicated primary antibody, followed by secondary antibody conjugated with horseradish peroxidase. Protein bands were detected using an enhanced chemiluminescence Western blotting detection kit and then exposed to X-ray film.

\section{Statistical analysis}

Results were represented as the mean \pm SE. Comparison between groups was made by ANOVA and analyzed by post-hoc test. Differences of $p<0.05$ were considered to be statistical significant.

\section{RESULTS AND DISCUSSION}

\section{Effect of pectinase on ginsenosides profile}

Ginseng is a well-known medicinal plant widely used in oriental societies as one of the most valuable medicines. Ginseng has a glucose lowering effect probably due to increasing insulin secretion, ameliorating insulin resistance or both. However, anti-diabetic efficacy of ginseng is not remarkable and often ambiguous, and thus most of physician would hesitate to recommend the ginseng to diabetic patients as a supplement. Therefore, in a series of investigations to develop anti-diabetic agent with higher efficacy, ginseng radix was processed with pectinase enzymes. When ginsenoside profile was analyzed by ultra performance liquid chromatography, ginseng radix and GINST showed quiet different profiles and therefore differential effects were expected. As shown in Fig. 1, the saponin peaks in untreated ginseng radix (ginsenoside $R b_{1}, R c, R_{2}$ and $R d$ ) were decreased during the enzyme treatment. One day after pectinase treatment, these four ginsenosides were difficult to identify in the chromatogram. On the other hand, IH-901 was appeared during the enzyme process (Fig. 1B). IH-901 is a final metabolite of ginsenoside and may be one of active components for pharmacological activity of GINST. Although Panax ginseng is known to have anti-diabetic activity, the active ingredient is not yet fully identified. Recently, we reported that IH-901 is the one to decrease the fasting blood glucose levels in C57BL/KsJ db/db mice via enhancing insulin secretion and improving insulin resistance $[13,17]$. Having these results we attempt to explore whether GINST shows anti-diabetic effect in ICR mice fed a high fat diet [18,19].
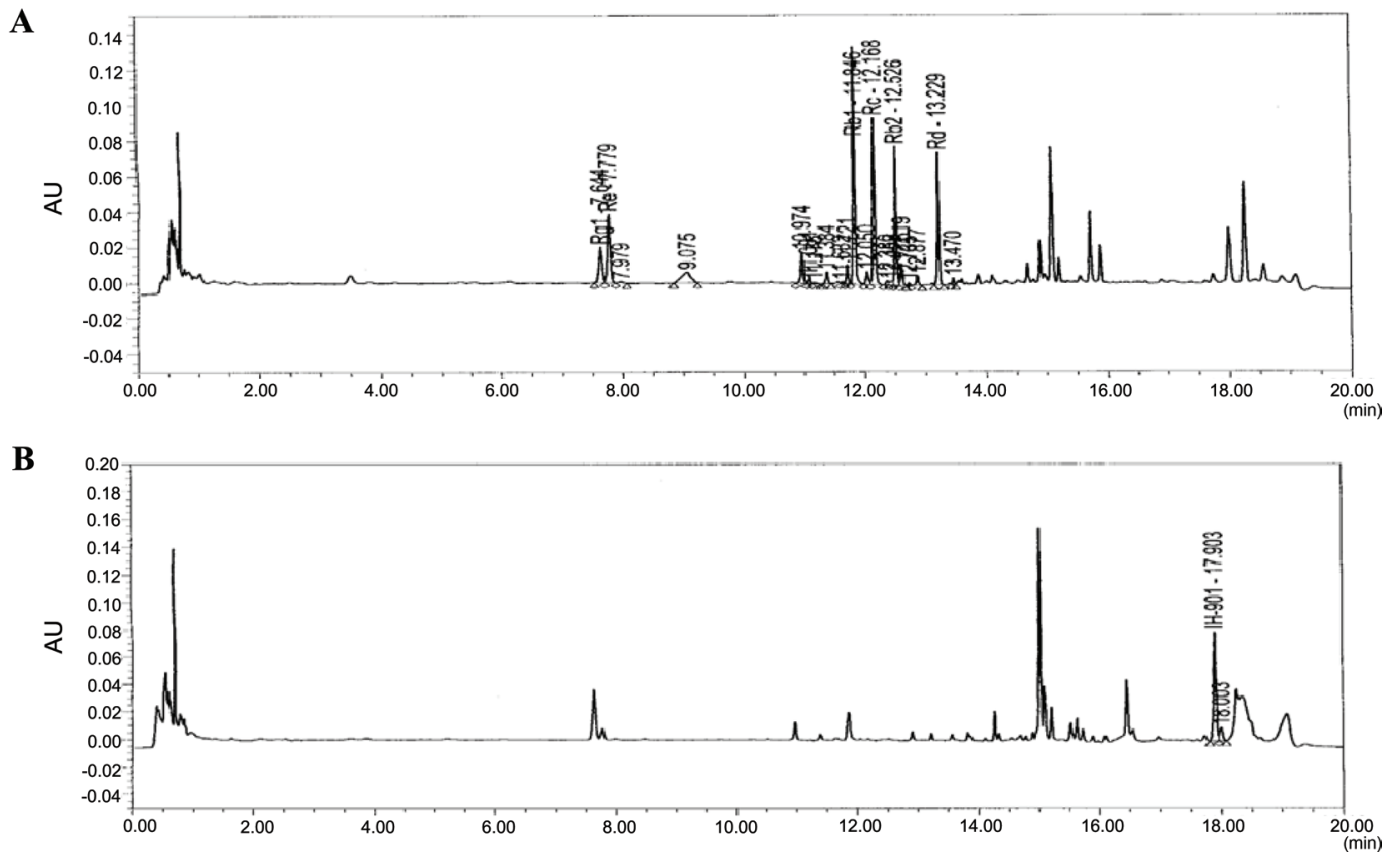

Fig. 1. Ultra performance liquid chromatography profiles of ginseng radix (A) and pectinase-processed ginseng radix (B). 


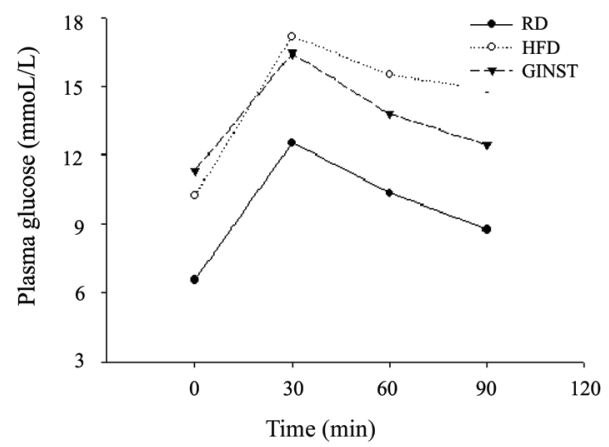

B

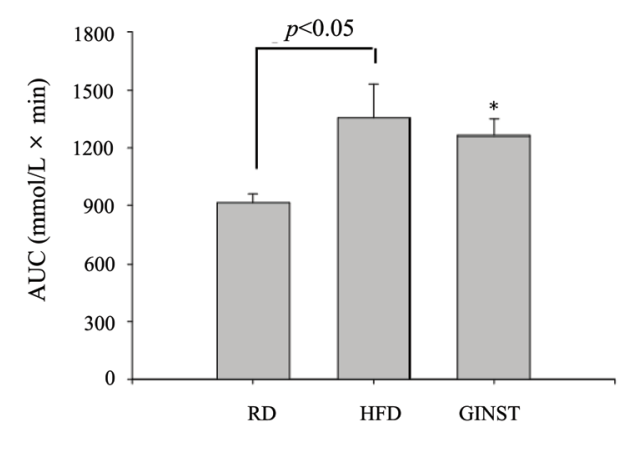

Fig. 2. Plasma glucose responses to an oral glucose challenge $(1.5 \mathrm{~g} / \mathrm{kg})$ after $12 \mathrm{~h}$ food deprivation in ICR mice. Plasma glucose response to an oral glucose challenge (A), and the area under the curve of plasma glucose concentration versus time (B). Values are mean $\pm S E(n=5)$ and ${ }^{*} p<0.05$ compared to high fat diet control group. RD, regular diet; HFD, high fat diet; GINST, pectinase-processed ginseng radix.

Table 1. Effects of pectinase-processed ginseng radix (GINST) on body weight and metabolic parameters

\begin{tabular}{lrcc}
\hline & \multicolumn{1}{c}{ RD } & \multicolumn{1}{c}{ HFD } & \multicolumn{1}{c}{ GINST } \\
\hline Body weight $(\mathrm{g})$ & $38.16 \pm 1.3$ & $43.2 \pm 1.4^{\# \#}$ & $41.2 \pm 1.6^{*}$ \\
Glucose $(\mathrm{mM})$ & $6.3 \pm 0.5$ & $15.2 \pm 3.4^{\# \#}$ & $6.4 \pm 1.3^{* *}$ \\
Insulin (U/ml) & $18.7 \pm 1.4$ & $33.3 \pm 2.4^{\# \#}$ & $23.0 \pm 2.9^{* *}$ \\
HOMA-IR & $5.3 \pm 0.7$ & $22.6 \pm 5.0^{\# \#}$ & $7.2 \pm 2.2^{* *}$
\end{tabular}

Data are mean \pm SE $(n=5)$. Homeostasis model assessment was used to calculate an index of insulin resistance as insulin $(\mu \mathrm{U} / \mathrm{mL}) \times$ glucose $(\mathrm{mM}) / 22.5$. $" p<0.05$ and $" p<0.01$ compared to high fat diet (HFD) control group. ${ }^{\#} p<0.01$ compared to regular diet (RD) control group

\section{Effects of GINST on hyperglycemia and insulin re- sistance induced by high fat diet}

To determine the effect of multiple oral administration of GINST on glucose tolerance, Oral glucose tolerance test was carried out at the end of the experiment. As shown in Fig. 2, glucose challenge significantly increased the blood glucose levels in the HFD control group, whereas GINST-treated group significantly suppressed the blood glucose levels from rising during $90 \mathrm{~min}$ after glucose challenge (Fig. 2A). Compared to the HFD control group, area under the curve was reduced by $7 \%$ $(p<0.05)$ (Fig. 2B). Body weight and metabolic parameters related to diabetes are shown in Table 1, body weight was increased by $11.3 \%$ in the HFD control group compared to the RD group. When compared to HFD control group, final body weight was lowered by $4.6 \%$ in GINST group. Fasting plasma glucose, insulin and the insulin resistance index (HOMA-IR) [20] levels in GINST group were significantly decreased by $57.8 \%(p<0.05), 30.9 \%$ $(p<0.01)$, and $68.1 \%(p<0.01)$, respectively, compared to the HFD control group (Table 1). Histological analysis of the pancreas from the HFD control group revealed a degeneration of islets, whereas mice treated with GINST preserved the islets architecture (Fig. 3A). In addition, insulin contents in GINST-treated group were restored when compared to the HFD control group (Fig. 3B). These results suggest that GINST may have beneficial effects on hyperglycemia and insulin resistance induced by HFD, and IH-901 might be responsible for these effects.

\section{Effects of GINST on protein expression of AMPK and GLUT4}

Resistance to the insulin action in skeletal muscle is a major risk factor in type 2 diabetes mellitus [5,21]. Skeletal muscle plays a crucial role in maintaining systemic glucose metabolism, accounting for $85 \%$ of whole body insulin-stimulated glucose uptake [22]. In skeletal muscle, insulin stimulates glucose uptake by increasing the translocation of glucose transport molecules, mainly GLUT4, from intracellular vesicles to the cell surface [23]. Therefore, elucidation of the signaling pathways governing contraction-induced increases in skeletal muscle glucose uptake may provide new pharmacological targets for the treatment of individuals with type 2 diabetes. AMPK is one of the most important factors for cellular energy balance and is recognized as a potential therapeutic target in the prevention and treatment of type 2 diabetes [24-26]. AMPK is emerging as a signaling intermediary that controls the use of glucose and fatty acids in skeletal muscle [27,28]. Increased GLUT4 expression or translocation to the plasma membrane can be regulated by the activation of AMPK through an insulinindependent mechanism [29]. Thus, in the present study, we examined whether GINST activates AMPK through phophorylation in the skeletal muscle. As shown in Fig. 4, GINST stimulates the phosphorylation of AMPK in the 
Con

$\mathbf{A}$

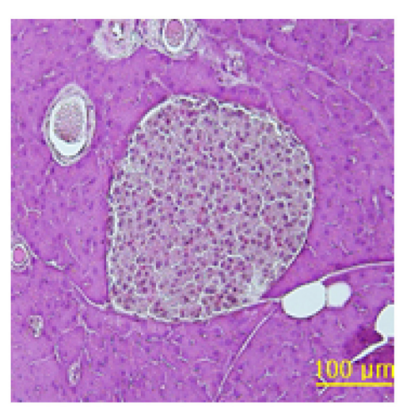

B

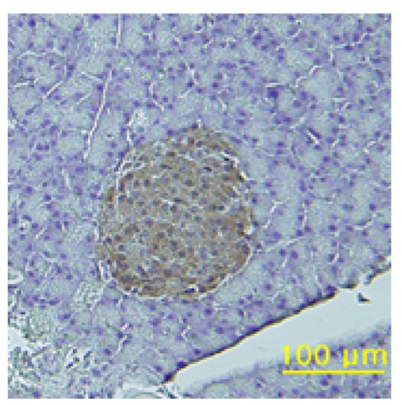

HFD
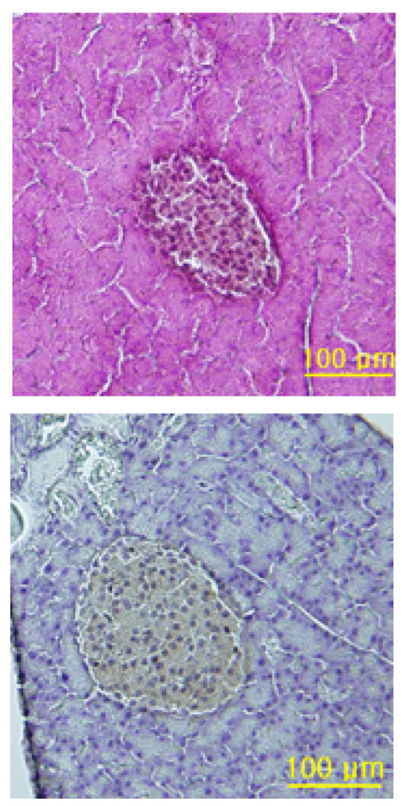

GINST
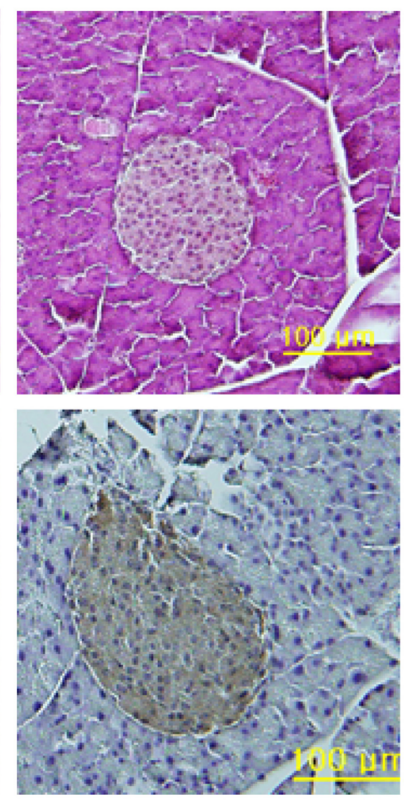

Fig. 3. Effect of pectinase-processed ginseng radix on pancreas morphology. Panel A represents H\&E staining and panel B represents insulin immunostaining (×200). Con, control; HFD, high fat diet; GINST, pectinase-processed ginseng radix.

$\mathbf{A}$

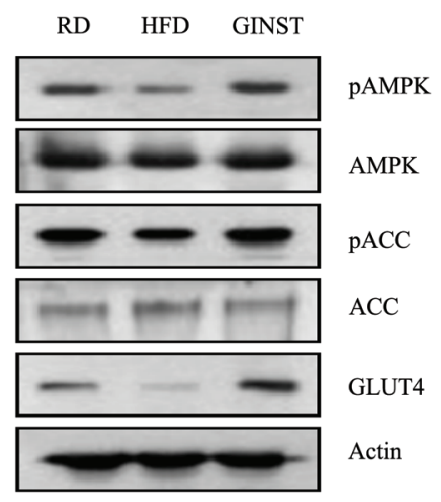

B

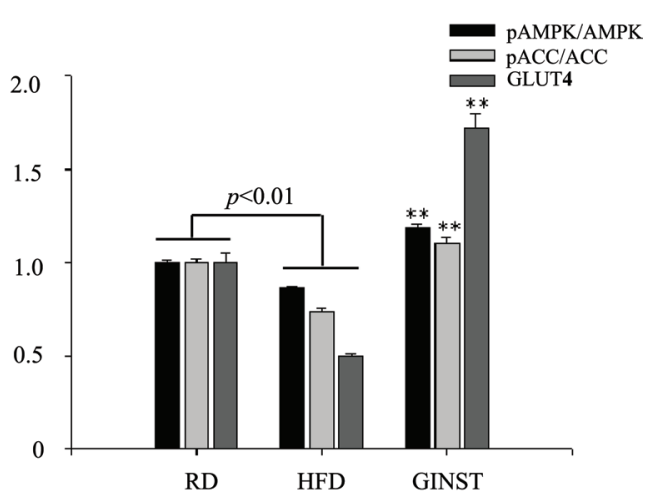

Fig. 4. Effects of pectinase-processed ginseng radix on pAMPK, pACC, and GLUT4 protein expression measured via Western blot analyses (A) and their densitometric analyses (B). RD, regular diet; HFD, high fat diet; GINST, pectinase-processed ginseng radix.

skeletal muscle. GLUT4 protein expression in the skeletal muscle was also markedly enhanced in GINST-treated group. Taken together, these results suggest that GINST lowered plasma glucose level through ameliorating insulin resistance via AMPK signal pathway in the skeletal muscle of high fat diet-fed ICR mice, and GINST can be a health functional food or therapeutic agent for type 2 diabetic patients.

\section{ACKNOWLEDGEMENTS}

This work was supported by a grant from the Kyung
Hee University post-doctoral fellowship in 2010 (KHU20100424).

\section{REFERENCES}

1. Liao Z, Chen X, Wu M. Antidiabetic effect of flavones from Cirsium japonicum DC in diabetic rats. Arch Pharm Res 2010;33:353-362.

2. Zhu CF, Peng HB, Liu GQ, Zhang F, Li Y. Beneficial effects of oligopeptides from marine salmon skin in a rat model of type 2 diabetes. Nutrition 2010;26:1014-1020.

3. Gabir MM, Hanson RL, Dabelea D, Imperatore G, Rou- 
main J, Bennett PH, Knowler WC. Plasma glucose and prediction of microvascular disease and mortality: evaluation of 1997 American Diabetes Association and 1999 World Health Organization criteria for diagnosis of diabetes. Diabetes Care 2000;23:1113-1118.

4. Wang ZQ, Zhang XH, Yu Y, Poulev A, Ribnicky D, Floyd ZE, Cefalu WT. Bioactives from bitter melon enhance insulin signaling and modulate acyl carnitine content in skeletal muscle in high-fat diet-fed mice. J Nutr Biochem 2011; Epub ahead of print.

5. Abdul-Ghani MA, DeFronzo RA. Pathogenesis of insulin resistance in skeletal muscle. J Biomed Biotechnol 2010;2010:476279.

6. Shaw RJ, Lamia KA, Vasquez D, Koo SH, Bardeesy N, Depinho RA, Montminy M, Cantley LC. The kinase LKB1 mediates glucose homeostasis in liver and therapeutic effects of metformin. Science 2005;310:16421646.

7. Baur JA, Pearson KJ, Price NL, Jamieson HA, Lerin C, Kalra A, Prabhu VV, Allard JS, Lopez-Lluch G, Lewis K et al. Resveratrol improves health and survival of mice on a high-calorie diet. Nature 2006;444:337-342.

8. Luo JZ, Luo L. Ginseng on hyperglycemia: effects and mechanisms. Evid Based Complement Alternat Med 2009;6:423-427.

9. Huang J, Tang XH, Ikejima T, Sun XJ, Wang XB, Xi $\mathrm{RG}, \mathrm{Wu}$ LJ. A new triterpenoid from Panax ginseng exhibits cytotoxicity through p53 and the caspase signaling pathway in the HepG2 cell line. Arch Pharm Res 2008;31:323-329.

10. Park EK, Shin YW, Lee HU, Kim SS, Lee YC, Lee BY, Kim DH. Inhibitory effect of ginsenoside $\mathrm{Rb}_{1}$ and compound $\mathrm{K}$ on $\mathrm{NO}$ and prostaglandin $\mathrm{E} 2$ biosyntheses of RAW264.7 cells induced by lipopolysaccharide. Biol Pharm Bull 2005;28:652-656.

11. Zhang D, Yasuda T, Yu Y, Zheng P, Kawabata T, Ma Y, Okada S. Ginseng extract scavenges hydroxyl radical and protects unsaturated fatty acids from decomposition caused by iron-mediated lipid peroxidation. Free Radic Biol Med 1996;20:145-150.

12. Chang Y, Huang WJ, Tien LT, Wang SJ. Ginsenosides $\mathrm{Rg}_{1}$ and $\mathrm{Rb}_{1}$ enhance glutamate release through activation of protein kinase A in rat cerebrocortical nerve terminals (synaptosomes). Eur J Pharmacol 2008;578:28-36.

13. Han GC, Ko SK, Sung JH, Chung SH. Compound K enhances insulin secretion with beneficial metabolic effects in db/db mice. J Agric Food Chem 2007;55:10641-10648.

14. Lee WK, Kao ST, Liu IM, Cheng JT. Ginsenoside $\mathrm{Rh}_{2}$ is one of the active principles of Panax ginseng root to improve insulin sensitivity in fructose-rich chow-fed rats.
Horm Metab Res 2007;39:347-354.

15. Park MW, Ha J, Chung SH. 20(S)-ginsenoside $\mathrm{Rg}_{3}$ enhances glucose-stimulated insulin secretion and activates AMPK. Biol Pharm Bull 2008;31:748-751.

16. Zhang Z, Li X, Lv W, Yang Y, Gao H, Yang J, Shen Y, Ning G. Ginsenoside Re reduces insulin resistance through inhibition of c-Jun NH2-terminal kinase and nuclear factor-kappaB. Mol Endocrinol 2008;22:186-195.

17. Yuan HD, Kim SJ, Chung SH. Beneficial effects of IH901 on glucose and lipid metabolisms via activating adenosine monophosphate-activated protein kinase and phosphatidylinositol-3 kinase pathways. Metabolism 2011;60:43-51.

18. Kusakabe T, Tanioka H, Ebihara K, Hirata M, Miyamoto L, Miyanaga F, Hige H, Aotani D, Fujisawa T, Masuzaki $\mathrm{H}$ et al. Beneficial effects of leptin on glycaemic and lipid control in a mouse model of type 2 diabetes with increased adiposity induced by streptozotocin and a highfat diet. Diabetologia 2009;52:675-683.

19. Sone H, Kagawa Y. Pancreatic beta cell senescence contributes to the pathogenesis of type 2 diabetes in high-fat diet-induced diabetic mice. Diabetologia 2005;48:58-67.

20. Matthews DR, Hosker JP, Rudenski AS, Naylor BA, Treacher DF, Turner RC. Homeostasis model assessment: insulin resistance and beta-cell function from fasting plasma glucose and insulin concentrations in man. Diabetologia 1985;28:412-419.

21. Choi K, Kim YB. Molecular mechanism of insulin resistance in obesity and type 2 diabetes. Korean J Intern Med 2010;25:119-129.

22. DeFronzo RA, Jacot E, Jequier E, Maeder E, Wahren J, Felber JP. The effect of insulin on the disposal of intravenous glucose. Results from indirect calorimetry and hepatic and femoral venous catheterization. Diabetes 1981; 30:1000-1007.

23. Peppa M, Koliaki C, Nikolopoulos P, Raptis SA. Skeletal muscle insulin resistance in endocrine disease. J Biomed Biotechnol 2010;2010:527850.

24. Hardie DG. Role of AMP-activated protein kinase in the metabolic syndrome and in heart disease. FEBS Lett 2008;582:81-89.

25. Misra P, Chakrabarti R. The role of AMP kinase in diabetes. Indian J Med Res 2007;125:389-398.

26. Hwang JT, Kwon DY, Yoon SH. AMP-activated protein kinase: a potential target for the diseases prevention by natural occurring polyphenols. N Biotechnol 2009;26:1722.

27. Winder WW, Hardie DG. AMP-activated protein kinase, a metabolic master switch: possible roles in type 2 diabetes. Am J Physiol 1999;277(1 Pt 1):E1-E10. 
28. Egawa T, Hamada T, Ma X, Karaike K, Kameda N, Masuda S, Iwanaka N, Hayashi T. Caffeine activates preferentially $\alpha 1$-isoform of 5'AMP-activated protein kinase in rat skeletal muscle. Acta Physiol (Oxf) 2011;201:227238.
29. Takikawa M, Inoue S, Horio F, Tsuda T. Dietary anthocyanin-rich bilberry extract ameliorates hyperglycemia and insulin sensitivity via activation of AMP-activated protein kinase in diabetic mice. J Nutr 2010;140:527-533. 